\title{
Harmonic operators: the dual perspective
}

\author{
Matthias Neufang* $\quad$ Volker Runde ${ }^{\dagger}$
}

\begin{abstract}
The study of harmonic functions on a locally compact group $G$ has recently been transferred to a "non-commutative" setting in two different directions: C.-H. Chu and A. T.-M. Lau replaced the algebra $L^{\infty}(G)$ by the group von Neumann algebra $\operatorname{VN}(G)$ and the convolution action of a probability measure $\mu$ on $L^{\infty}(G)$ by the canonical action of a positive definite function $\sigma$ on $\operatorname{VN}(G)$; on the other hand, W. Jaworski and the first-named author replaced $L^{\infty}(G)$ by $\mathcal{B}\left(L^{2}(G)\right)$ to which the convolution action by $\mu$ can be extended in a natural way. We establish a link between both approaches. The action of $\sigma$ on $\operatorname{VN}(G)$ can be extended to $\mathcal{B}\left(L^{2}(G)\right)$. We study the corresponding space $\tilde{\mathcal{H}}_{\sigma}$ of " $\sigma$-harmonic operators", i.e., fixed points in $\mathcal{B}\left(L^{2}(G)\right)$ under the action of $\sigma$. We show, under mild conditions on either $\sigma$ or $G$, that $\tilde{\mathcal{H}}_{\sigma}$ is in fact a von Neumann subalgebra of $\mathcal{B}\left(L^{2}(G)\right)$. Our investigation of $\tilde{\mathcal{H}}_{\sigma}$ relies, in particular, on a notion of support for an arbitrary operator in $\mathcal{B}\left(L^{2}(G)\right)$ that extends Eymard's definition for elements of $\operatorname{VN}(G)$. Finally, we present an approach to $\tilde{\mathcal{H}}_{\sigma}$ via ideals in $\mathcal{T}\left(L^{2}(G)\right)$-where $\mathcal{T}\left(L^{2}(G)\right)$ denotes the trace class operators on $L^{2}(G)$, but equipped with a product different from composition-, as it was pioneered for harmonic functions by G. A. Willis.
\end{abstract}

Keywords: locally compact group; positive definite function; Fourier algebra; completely bounded multiplier; harmonic operator.

2000 Mathematics Subject Classification: Primary 22D99; Secondary 22D20, 22D25, 22D35, 43A35, 46L07, 46L10, 47L50.

\section{Introduction}

Let $G$ be a locally compact group, and let $\mu$ be a probability measure on $G$ whose support generates $G$. A function $\phi \in L^{\infty}(G)$ is called $\mu$-harmonic if $\mu * \phi=\phi$. Harmonic functions play a crucial rôle for the investigation of random walks on locally compact groups and are extensively studied (see [Aze and Fur, for example). The collection of all $\mu$-harmonic functions is denoted by $\mathcal{H}_{\mu}$; it is easily seen to be a $w^{*}$-closed subspace of $L^{\infty}(G)$, but is

${ }^{*}$ Research supported by NSERC and the Mathematisches Forschungsinstitut Oberwolfach.

${ }^{\dagger}$ Research supported by NSERC and the Mathematisches Forschungsinstitut Oberwolfach. 
a subalgebra only if it consists of the constants alone. Nevertheless, $\mathcal{H}_{\mu}$ can be equipped with a product - different, of course, from the one in $L^{\infty}(G)$ - turning it into an abelian von Neumann algebra ( Aze $)$.

Recently, the notion of harmonicity has been "quantized" in two, seemingly entirely different directions.

One such quantization was introduced and studied by C.-H. Chu and A. T.-M. Lau in $\mathrm{C}-\mathrm{L}$. Their approach can be considered dual to the classical concept of a harmonic function. In Eym, P. Eymard introduced the so-called Fourier algebra $A(G)$ and Fourier-Stieltjes algebra $B(G)$ of $G$. If $G$ is abelian with dual group $\hat{G}$, the Fourier and Fourier-Stieltjes transforms, respectively, yield isometric isomorphisms $A(G) \cong L^{1}(\hat{G})$ and $B(G) \cong M(\hat{G})$. Instead of looking at harmonicity with respect to a probability measure, Chu and Lau consider harmonicity of functionals on $A(G)$, i.e., of elements of the group von Neumann algebra $\operatorname{VN}(G)$, with respect to a positive definite function $\sigma \in B(G)$ : an operator $T \in \operatorname{VN}(G)$ is said to be $\sigma$-harmonic if $\sigma \cdot T=T$, where - is the canonical module action of $B(G)$ on $\operatorname{VN}(G)$. The collection of all $\sigma$-harmonic functionals is denoted by $\mathcal{H}_{\sigma}$. Even though, this new theory seems to parallel the classical theory of harmonic functions on the surface, it is, in fact, strikingly different. For instance, $\mathcal{H}_{\sigma}$ is always a von Neumann subalgebra of $\mathrm{VN}(G)$ ( $\mathrm{C}-\mathrm{L}$, Remark 3.2.11]).

A completely different type of quantization was recently carried out by W. Jaworski and the first-named author $(\underline{\mathrm{J}-\mathrm{N}]})$. Starting point is the the result by F. Ghahramani (Gha ) that there is a natural isometric representation $\theta$ of the measure algebra $M(G)$ on $\mathcal{B}\left(L^{2}(G)\right)$, such that for $\mu \in M(G)$ and $\phi \in L^{\infty}(G)$-viewed as a multiplication operator on $L^{2}(G)$-we have $\theta(\mu) \phi=\mu * \phi$. Hence, the authors of [J-N] define an operator $T \in \mathcal{B}\left(L^{2}(G)\right)$ to be $\mu$-harmonic for a probability measure $\mu$ if $\theta(\mu)(T)=T$. The collection of all $\mu$-harmonic operators is denoted by $\tilde{\mathcal{H}}_{\mu}$. Like $\mathcal{H}_{\mu}$, the space $\tilde{\mathcal{H}}_{\mu}$ is a von Neumann algebra, but with a product usually different from the one in $\mathcal{B}\left(L^{2}(G)\right)$; in fact, $\tilde{\mathcal{H}}_{\mu}$ can be described as the crossed product of $\mathcal{H}_{\mu}$ with $G$, where the action of $G$ on $\mathcal{H}_{\mu}$ is given by left translation ( $\underline{\mathrm{J}-\mathrm{N}}$, Proposition 6.3]).

In the present paper, we extend Chu's and Lau's notion of $\sigma$-harmonicity from $\operatorname{VN}(G)$ to $\mathcal{B}\left(L^{2}(G)\right)$ in a way that parallels the extension of $\mu$-harmonicity from $L^{\infty}(G)$ to $\mathcal{B}\left(L^{2}(G)\right)$ in [J-N].

As the predual space of the operator algebra $\mathrm{VN}(G)$, the Fourier algebra carries a canonical operator space structure. (For the theory of operator spaces, we refer to $[\mathrm{E}-\mathrm{R}]$.) By $\mathcal{M}_{\mathrm{cb}}(A(G))$, we denote the completely bounded multipliers of $A(G)$, i.e., those functions on $G$ that induce completely bounded multiplication operators on $A(G)$. Obviously, $\mathcal{M}_{\mathrm{cb}}(A(G))$ is a commutative Banach algebra, and it contains $B(G)$ (with equality if and only if $G$ is amenable). In $[\mathrm{N}-\mathrm{R}-\mathrm{S}$, the first-named author, Z.-J. Ruan, and N. Spronk constructed a representation $\hat{\theta}$ of $\mathcal{M}_{\mathrm{cb}}(A(G))$ on $\mathcal{B}\left(L^{2}(G)\right)$ which displays a perfect dual- 
ity with Ghahramani's representation of $M(G)$ (for details, see $[\mathrm{N}-\mathrm{R}-\mathrm{S}]$ ); in particular, it extends the action of $B(G)$ on $\operatorname{VN}(G)$ to $\mathcal{B}\left(L^{2}(G)\right)$. For $\sigma \in \mathcal{M}_{\mathrm{cb}}(A(G))$, it is then natural to define $T \in \mathcal{B}\left(L^{2}(G)\right)$ to be $\sigma$-harmonic if $\hat{\theta}(\sigma)(T)=T$. We denote the collection of all $\sigma$-harmonic operators by $\tilde{\mathcal{H}}_{\sigma}$.

The aim of this paper is to explore the connections between this setting and the two quantizations from $[\mathrm{C}-\mathrm{L}]$ and $[\mathrm{J}-\mathrm{N}]$. For instance, one of our main results is that-under very mild hypotheses which are always satisfied if $G$ is amenable or the free group in two generators - $\tilde{\mathcal{H}}_{\sigma}$ is a von Neumann subalgebra of $\mathcal{B}\left(L^{2}(G)\right)$, namely the von Neumann subalgebra of $\mathcal{B}\left(L^{2}(G)\right)$ generated by $\mathcal{H}_{\sigma}$ and $L^{\infty}(G)$.

The paper is organized as follows.

First, we fix our notation and terminology and review a few basic facts about harmonic functions (see $[\mathrm{J}-\mathrm{N}]$ ) and harmonic functionals in $\mathrm{VN}(G)$ (as introduced and studied in C-L]). We also recall results from $\mathbb{N}-\mathrm{R}-\mathrm{S}$, on the representation of $\mathcal{M}_{\mathrm{cb}}(A(G))$ on $\mathcal{B}\left(L^{2}(G)\right)$.

Section 2 gives a characterization of adaptedness in terms of the Fourier-Stieltjes transform (in the framework of locally compact abelian groups). This motivates our definition of the dual notion of adaptedness for positive definite functions.

In Section 3, we introduce the support of an arbitrary (bounded linear) operator on $L^{2}(G)$ such that it generalizes Eymard's corresponding notion for elements of $\operatorname{VN}(G)$. In order to prepare the ground for our main results, we investigate the properties of our notion of support in detail.

Those main results are contained in Section 4. Using the representation of $\mathcal{M}_{\mathrm{cb}}(A(G))$ from $[\mathrm{N}-\mathrm{R}-\mathrm{S}$, we naturally extend the notion of a $\sigma$-harmonic functional in $\mathrm{VN}(G)([\mathrm{C}-\mathrm{L}])$ to the one of a $\sigma$-harmonic operator in $\mathcal{B}\left(L^{2}(G)\right)$, where $\sigma$ is a positive definite function on $G$. Assuming either a very mild condition on $G$ - the approximation property $([\underline{\mathrm{H}}-\mathrm{K}]$ ) - or that $\sigma$ belongs to $A(G)$, we show that the space of $\sigma$-harmonic operators $\tilde{\mathcal{H}}_{\sigma}$ is always a von Neumann subalgebra of $\mathcal{B}\left(L^{2}(G)\right)$ and, in fact, precisely the von Neumann algebra generated by $L^{\infty}(G)$ and the algebra $\mathcal{H}_{\sigma}$ of $\sigma$-harmonic functionals in $\operatorname{VN}(G)$. This may be viewed as a result "dual" to the corresponding characterization (see [J-N]) of the von Neumann algebra of $\mu$-harmonic operators in $\mathcal{B}\left(L^{2}(G)\right)$ as a crossed product over the algebra of $\mu$-harmonic functions in $L^{\infty}(G)$, where $\mu$ is a probability measure on $G$. Our notion of support for an arbitrary operator on $L^{2}(G)$ allows for an alternative characterization of $\tilde{\mathcal{H}}_{\sigma}$-at least if $G$ has the approximation property-, namely as the operators in $\mathcal{B}\left(L^{2}(G)\right)$ with support contained in the subgroup $G_{\sigma}=\sigma^{-1}(\{1\})$ of $G$.

Finally, in Section 5 we present an approach to harmonic operators in $\mathcal{B}\left(L^{2}(G)\right)$ via ideals in the predual $\mathcal{T}\left(L^{2}(G)\right)$ in the spirit of Wil] (see also [J-N Section 3]). This makes it necessary to endow the space $\mathcal{T}\left(L^{2}(G)\right)$ with a product very different from the composition of operators; this product arises naturally when when one regards $\mathcal{B}\left(L^{2}(G)\right)$ 
as a Hopf-von Neumann algebra, with a co-multiplication naturally extending the one of $\mathrm{VN}(G)$ (see $[\mathrm{N}-\mathrm{R}-\mathrm{S}],[\mathrm{P}-\mathrm{R}]$, and $[\mathrm{J}-\mathrm{N}]$ ).

\section{Acknowledgments}

Most of this paper was written during an RiP (= "Research in Paradise") stay by the authors at the Mathematisches Forschungsinstitut Oberwolfach in May 2005. We acknowledge this support and the uniquely stimulating atmosphere at Oberwolfach with gratitude.

We would also like to thank Tony Lau for helping with the proof of Lemma 4.4 -in particular, for bringing [Lau] to our attention - and Zhiguo Hu for catching some oversights in an earlier version of this paper. Finally, thanks are due to the referee for his helpful suggestions.

\section{Preliminaries}

Throughout this section, let $G$ denote a locally compact group equipped with left Haar measure. We follow standard notation and terminology of abstract harmonic analysis as, e.g., in $[\mathrm{H}-\mathrm{R}]$. For $p \in[1, \infty]$, we denote the corresponding $L^{p}$-space with respect to left Haar measure on $G$ by $L^{p}(G)$. By $M(G)$, we denote the algebra of all complex, regular Borel measures; $M^{1}(G)$ stands for the probability measures in $M(G)$. We note that $L^{\infty}(G)$ is a von Neumann algebra acting naturally on $L^{2}(G)$ as multiplication operators: for $\phi \in L^{\infty}(G)$, let $M_{\phi} \in \mathcal{B}\left(L^{2}(G)\right)$ be given by $\left(M_{\phi} \xi\right)(x)=\phi(x) \xi(x)$ for $\xi \in L^{2}(G)$ and $x \in G$. (For the sake of notational simplicity, we shall sometimes write $\phi$ and $M_{\phi}$ interchangeably.)

The Banach algebra $M(G)$ acts on $L^{p}(G)$ for $p \in[1, \infty]$ via convolution from the left. For $\mu \in M(G)$, we define $\phi \in L^{\infty}(G)$ as $\mu$-harmonic if $\mu * \phi=\phi$ and set

$$
\mathcal{H}_{\mu}:=\left\{\phi \in L^{\infty}(G): \phi \text { is } \mu \text {-harmonic }\right\}
$$

(For the motivation for the name " $\mu$-harmonic function", see the introduction of $[\mathrm{C}-\mathrm{L}]$ ). Usually, $\mu$-harmonic functions are considered only for $\mu \in M^{1}(G)$. To avoid pathologies, we shall assume $\mu$ to be adapted, i.e., $\langle\operatorname{supp} \mu\rangle$, the closed subgroup of $G$ generated by the support of $\mu$, is all of $G$. For abelian $G$ and adapted $\mu \in M^{1}(G)$, the classical ChoquetDeny theorem asserts that $\mathcal{H}_{\mu}$ consists only of the constant functions. For a general locally compact group $G$, a measure $\mu \in M^{1}(G)$ such that $\mathcal{H}_{\mu} \cong \mathbb{C}$ exists if and only if $G$ is amenable and $\sigma$-compact $\left(\underline{\mathrm{C}-\mathrm{L}}\right.$, Proposition 2.1.3]). Even though $\mathcal{H}_{\mu}$ is not a von Neumann subalgebra of $L^{\infty}(G)$ - except if $\mathcal{H}_{\mu} \cong \mathbb{C}-$, there is a natural product on $\mathcal{H}_{\mu}$ turning it into an abelian von Neumann algebra in its own right: given $f, g \in \mathcal{H}_{\mu}$, one can 
show that the limit

$$
w^{*}-\lim _{n} \int_{G} \rho(x)(f g) d \mu^{* n}(x)
$$

exists in $L^{\infty}(G)$ and is an explicit formula for the multiplication in $\mathcal{H}_{\mu}$ (here, $\mu^{* n}$ stands for the $n$-th convolution power of $\mu$, and $\rho(x)$ denotes right translation by $x$, i.e., $(\rho(x) \phi)(y):=$ $\phi(y x)$ for $\phi: G \rightarrow \mathbb{C}$ and $x, y \in G)$. For the classical theory of random walks and harmonic functions, see $[\mathrm{Aze}$, $\mathrm{Nev}$, and $[\mathrm{Rev}$, for example.

For any $\phi: G \rightarrow \mathbb{C}$ and $x \in G$, we define $\lambda(x) \phi: G \rightarrow \mathbb{C}$ by letting $(\lambda(x) \phi)(y):=$ $\phi\left(x^{-1} y\right)$ for $x \in G$. For fixed $x \in G$, the map $L^{2}(G) \ni \xi \mapsto \lambda(x) \xi$ is a unitary operator on $L^{2}(G)$, and

$$
\lambda: G \rightarrow \mathcal{B}\left(L^{2}(G)\right), \quad x \mapsto \lambda(x)
$$

is a unitary representation of $G$ on $L^{2}(G)$, the left regular representation of $G$. The group von Neumann algebra $\operatorname{VN}(G)$ of $G$ is defined as $\operatorname{VN}(G):=\lambda(G)^{\prime \prime}$. The Fourier algebra $A(G)$ of $G$-introduced by P. Eymard in Eym - is the (unique) predual of $\operatorname{VN}(G)$.

As any operator algebra, $\mathrm{VN}(G)$ is a concrete operator space. (For the theory of operator spaces, our reference is $[\mathrm{E}-\mathrm{R}])$. Since the category of operator spaces allows for a natural duality theory, the dual space $\operatorname{VN}(G)^{*}$ - and thus its subspace $A(G)$ - is an operator space in a canonical manner. In particular, we may speak of completely bounded maps on $A(G)$. (Following [E-R], we denote the completely bounded maps on an operator space $E$ by $\mathcal{C B}(E)$.) A multiplier of $A(G)$ is a function $\sigma$ on $G$ such that $\sigma A(G) \subset A(G)$; a multiplier is completely bounded if the corresponding multiplication operator is completely bounded. The collection of all completely bounded multipliers is denoted by $\mathcal{M}_{\mathrm{cb}}(A(G))$. It naturally inherits an operator space structure from $\mathcal{C B}(A(G))$ which turns it into a commutative, completely contractive Banach algebra, i.e., a Banach algebra which is an operator space such that multiplication is completely contractive. An important subalgebra of $\mathcal{M}_{\mathrm{cb}}(A(G))$ is the Fourier-Stieltjes algebra $B(G)$ of $G$ (introduced also in Eym). It consists of all functions of the form $G \ni x \mapsto\langle\pi(x) \xi, \eta\rangle$, where $\pi$ is a (always strongly continuous) unitary representation of $G$ on a Hilbert space $\mathfrak{H}$ and $\xi, \eta \in \mathfrak{H}$. It can be identified with the dual space of the full group $C^{*}$-algebra $C^{*}(G)$, i.e., of the enveloping $C^{*}$-algebra of $L^{1}(G)$, and thus also has a canonical operator space structure. It contains $A(G)$ as a closed ideal and thus canonically embeds into $\mathcal{M}_{\mathrm{cb}}(A(G))$; this embedding is always completely contractive, but is an isometric isomorphism if and only if $G$ is amenable. For more details and references to the original literature, see $\mathrm{Spr}$.

It is immediate from the definition of $\mathcal{M}_{\mathrm{cb}}(A(G))$ that $A(G)$ is a completely contractive Banach $\mathcal{M}_{\mathrm{cb}}(A(G))$-module through pointwise multiplication. Consequently, $\mathrm{VN}(G)$ carries a dual $\mathcal{M}_{\mathrm{cb}}(A(G))$-module structure, namely

$$
\langle\phi, \sigma \cdot T\rangle:=\langle\phi \sigma, T\rangle \quad\left(\sigma \in \mathcal{M}_{\mathrm{cb}}(A(G)), T \in \mathrm{VN}(G), \phi \in A(G)\right) .
$$


Slightly generalizing the definition from $\mathrm{C}-\mathrm{L}$, we say that, for $\sigma \in \mathcal{M}_{\mathrm{cb}}(A(G))$, a von Neumann algebra element $T \in V(G)$, is $\sigma$-harmonic if $\sigma \cdot T=T$, and define

$$
\mathcal{H}_{\sigma}:=\{T \in \mathrm{VN}(G): T \text { is } \sigma \text {-harmonic }\} .
$$

(Chu and Lau consider $\mathcal{H}_{\sigma}$ only for $\sigma \in P^{1}(G)$, which denotes the positive definite functions of norm one in $B(G)$.) For abelian $G$ with dual group $\hat{G}$, the Fourier and Fourier-Stieltjes transforms, respectively, yield isometric isomorphism $A(G) \cong L^{1}(\hat{G})$ and $B(G) \cong M(\hat{G})$, and conjugation with the Plancherel transform yields that

$$
\mathcal{H}_{\mu} \cong \mathcal{H}_{\hat{\mu}} \quad(\mu \in M(G))
$$

Despite the formal analogies with harmonic functions, the harmonic functionals according to Chu and Lau display a strikingly different behavior: for $\sigma \in P^{1}(G)$, the set

$$
G_{\sigma}:=\{x \in G: \sigma(x)=1\}
$$

is a closed subgroup of $G$ such that $\mathcal{H}_{\sigma}=\lambda\left(G_{\sigma}\right)^{\prime \prime}$ ([C-L, Proposition 3.2.10]); in particular, $\mathcal{H}_{\sigma}$ is a von Neumann subalgebra of $\mathcal{B}\left(L^{2}(G)\right)$.

Finally, we require a construction from $[\mathrm{N}-\mathrm{R}-\mathrm{S}]$ : there the first-named author, Z.-J. Ruan, and N. Spronk define complete isometry $\hat{\theta}: \mathcal{M}_{\mathrm{cb}}(A(G)) \rightarrow \mathcal{C B}\left(\mathcal{B}\left(L^{2}(G)\right)\right)$ with the following properties:

- $\hat{\theta}$ is a unital, $w^{*}-w^{*}$-continuous algebra homomorphism;

- $\hat{\theta}\left(\mathcal{M}_{\mathrm{cb}}(A(G))\right)$ consists precisely of those normal operators in $\mathcal{C B}\left(\mathcal{B}\left(L^{2}(G)\right)\right)$ which are $L^{\infty}(G)$-bimodule homomorphisms and leave $\mathrm{VN}(G)$ invariant;

- for $\phi \in \mathcal{M}_{\mathrm{cb}}(A(G))$ and $T \in \mathrm{VN}(G)$, we have $\hat{\theta}(\phi)(T)=\phi \cdot T$, i.e., the action of $\mathcal{M}_{\mathrm{cb}}(A(G))$ on $\mathcal{B}\left(L^{2}(G)\right)$ induced by $\hat{\theta}$ extends the canonical one of $\mathcal{M}_{\mathrm{cb}}(A(G))$ on $\mathrm{VN}(G)$.

Under many aspects, $\hat{\theta}$ can be viewed as dual to Ghahramani's representation of $M(G)$ on $\mathcal{B}\left(L^{2}(G)\right)$ (for details, see Section 4 and $[\mathrm{N}-\mathrm{R}-\mathrm{S}]$ ).

For later use, we indicate how $\hat{\theta}$ is defined. Given $\phi \in \mathcal{M}_{\mathrm{cb}}(A(G))$, there are a Hilbert space $\mathfrak{H}$ and continuous functions $\boldsymbol{\xi}, \boldsymbol{\eta}: G \rightarrow \mathfrak{H}$ such that

$$
\phi\left(x^{-1} y\right)=\langle\boldsymbol{\xi}(y), \boldsymbol{\eta}(x)\rangle \quad(x, y \in G)
$$

(see [Jol] for an accessible proof). Let $\left(e_{i}\right)_{i \in \mathbb{I}}$ be an orthonormal basis, and define

$$
\phi_{i}(x):=\left\langle e_{i}, \boldsymbol{\eta}(x)\right\rangle \quad \text { and } \quad \psi_{i}(x):=\left\langle\boldsymbol{\xi}(x), e_{i}\right\rangle \quad(i \in \mathbb{I}, x \in G) .
$$

Then we have

$$
\hat{\theta}(\phi)(T):=\sum_{i \in \mathbb{I}} M_{\phi_{i}(x)} T M_{\psi_{i}(x)} .
$$

For more details, see $[\mathrm{N}-\mathrm{R}-\mathrm{S}$. 


\section{A dual characterization of adapted probability measures}

Let $G$ be a locally compact group, and let $\mu \in M^{1}(G)$. It is fair to say that almost nothing can be said about $\mathcal{H}_{\mu}$ unless $\mu$ is adapted. In order to have a dual notion of adaptedness, i.e., in the context of harmonic functionals as in $\mathrm{C}-\mathrm{L}$, we first characterize the adapted probability measures on a locally compact abelian group in terms of their Fourier-Stieltjes transforms.

Proposition 2.1. Let $G$ be a locally compact abelian group. Then the following are equivalent for $\mu \in M^{1}(G)$ :

(i) $\mu$ is adapted;

(ii) if $\gamma \in \hat{G}$ is such that $\left.\gamma\right|_{\operatorname{supp} \mu} \equiv 1$, then $\gamma=1$;

(iii) $\{\gamma \in \hat{G}: \hat{\mu}(\gamma)=1\}=\{1\}$.

Proof. (i) $\Longleftrightarrow$ (ii) and (iii) $\Longrightarrow$ (i) are straightforward.

(ii) $\Longrightarrow$ (iii): Let $\gamma \in \hat{G}$ be such that

$$
\hat{\mu}(\gamma)=\int_{G} \overline{\gamma(x)} d \mu(x)=1
$$

so that

$$
1=\overline{\hat{\mu}(\gamma)}=\overline{\int_{G} \overline{\gamma(x)} d \mu(x)}=\int_{G} \gamma(x) d \mu(x)
$$

and thus

$$
\int_{G} \operatorname{Re} \gamma(x) d \mu(x)=\frac{1}{2}\left(\int_{G} \overline{\gamma(x)} d \mu(x)+\int_{G} \gamma(x) d \mu(x)\right)=1 .
$$

Since $\gamma(G) \subset \mathbb{T}$, we have $(\operatorname{Re} \gamma)(G) \subset[-1,1]$, so that

$$
0=\int_{G} 1 d \mu(x)-\int_{G} \operatorname{Re} \gamma(x) d \mu(x)=\int_{\operatorname{supp} \mu} \underbrace{(1-\operatorname{Re} \gamma(x))}_{\geq 0} d \mu(x) .
$$

By the continuity of $\gamma$, this means that $\left.\operatorname{Re} \gamma\right|_{\operatorname{supp} \mu} \equiv 1$, thus $\left.\gamma\right|_{\operatorname{supp} \mu} \equiv 1$, and therefore $\gamma=1$ by (ii).

Using (11) and [C-L, Proposition 3.2.10], we obtain a dual approach to the ChoquetDeny theorem:

Corollary 2.2. Let $G$ be a locally compact abelian group, and let $\mu \in M^{1}(G)$ be adapted. Then $\mathcal{H}_{\mu} \cong \mathbb{C}$ holds.

In view of Proposition 2.1, we define for general locally compact groups:

Definition 2.3. Let $G$ be a locally compact group. Then we call $\sigma \in P^{1}(G)$ adapted if $G_{\sigma}=\{e\}$. 
Remark. In terms of Definition [2.3. Proposition 2.1] can be reformulated as follows: a probability measure on a locally compact abelian group is adapted if and only if its Fourier-Stieltjes transform is adapted.

It is well known that there is an adapted probability measure on a locally compact group $G$ if and only if $G$ is $\sigma$-compact. Since a locally compact abelian group is $\sigma$-compact if and only if its dual is first countable, it is immediate that there is an adapted positive definite function on such a group if and only if it is first countable.

The following proposition extends this to general locally compact groups:

Proposition 2.4. The following are equivalent for a locally compact group:

(i) there is an adapted $\sigma \in P^{1}(G)$;

(ii) $G$ is first countable;

(iii) there is an adapted $\sigma \in A(G) \cap P^{1}(G)$.

Proof. (i) $\Longrightarrow$ (ii): Let $\sigma \in P^{1}(G)$ be adapted, fix a compact neighborhood $U$ of $e$, and define, for $n \in \mathbb{N}$,

$$
U_{n}:=\left\{x \in U:|\sigma(x)-1|<\frac{1}{n}\right\} .
$$

From the continuity of $\sigma$, it is clear that $\left\{U_{n}: n \in \mathbb{N}\right\}$ consists of neighborhoods of $e$. Let $V$ be a neighborhood of $e$, and suppose without loss of generality that $V$ is open and contained in $U$. Since $\sigma$ is continuous and adapted, and since $U \backslash V$ is compact, we have that

$$
\epsilon_{0}:=\inf \{|\sigma(x)-1|: x \in U \backslash V\}>0 .
$$

Choose $n_{0} \in \mathbb{N}$ so large that $\frac{1}{n_{0}} \leq \epsilon_{0}$. It follows that $U_{n_{0}} \subset V$. Consequently, $\left\{U_{n}: n \in \mathbb{N}\right\}$ is a base of neighborhoods of $e$.

Through translation, we see that every point of $G$ has a countable base of neighborhoods.

(ii) $\Longrightarrow$ (iii): Let $\left\{U_{n}: n \in \mathbb{N}\right\}$ be a base of neighborhoods of $e$, and suppose without loss of generality that $U_{n+1} \subset U_{n}$ for $n \in \mathbb{N}$. For each $n \in \mathbb{N}$, there is $\sigma_{n} \in A(G) \cap P^{1}(G)$ with $\operatorname{supp} \sigma_{n} \subset U_{n}$. Define

$$
\sigma:=\sum_{n=1}^{\infty} \frac{1}{2^{n}} \sigma_{n}
$$

so that, clearly, $\sigma \in A(G) \cap P^{1}(G)$. Let $x \in G \backslash\{e\}$. Since $\left\{U_{n}: n \in \mathbb{N}\right\}$ is a base of neighborhoods of $e$, there is $n_{0} \in \mathbb{N}$ such that $x \notin U_{n_{0}}$ and thus $x \notin U_{n}$ for $n \geq n_{0}$. It follows that

$$
|\sigma(x)| \leq \sum_{n=1}^{n_{0}-1} \frac{1}{2^{n}}\left|\sigma_{n}(x)\right| \leq \sum_{n=1}^{n_{0}-1} \frac{1}{2^{n}}<1 .
$$

This proves (iii).

Finally, (iii) $\Longrightarrow$ (i) is trivial. 


\section{The support of an operator on $L^{2}(G)$}

In Eym, P. Eymard introduced the notion of support for elements of a group von Neumann algebra: if $G$ is a locally compact group and $T \in \mathrm{VN}(G)$, then the support $\operatorname{supp} T$ of $T$ is defined to consist of those $x \in G$ such that $\phi(x)=0$ for all $\phi \in A(G)$ with $\phi \cdot T=0$.

Using $\hat{\theta}$, this notion can naturally be extended to arbitrary operators on $L^{2}(G)$ :

Definition 3.1. Let $G$ be a locally compact group, and let $T \in \mathcal{B}\left(L^{2}(G)\right)$. Then the support $\operatorname{supp} T$ of $T$ is defined to be

$$
\operatorname{supp} T:=\{x \in G: \phi(x)=0 \text { for all } \phi \in A(G) \text { with } \hat{\theta}(\phi)(T)=0\} .
$$

Remarks. 1. For operators in $\operatorname{VN}(G)$, this notion of support coincides with the one from Eym.

2. The support of an operator is obviously a closed subset of $G$.

We first prove a few general assertions on the support of an operator.

Proposition 3.2. Let $G$ be a locally compact group. Then, for $\phi \in A(G)$ and $T \in$ $\mathcal{B}\left(L^{2}(G)\right)$, we have

$$
\operatorname{supp} \hat{\theta}(\phi)(T) \subseteq \operatorname{supp} \phi \cap \operatorname{supp} T .
$$

Proof. Fix $\phi \in A(G)$ and $T \in \mathcal{B}\left(L^{2}(G)\right)$.

Since $\hat{\theta}$ is multiplicative and $A(G)$ is commutative, it is straightforward that any $\psi \in A(G)$ with $\hat{\theta}(\psi)(T)=0$ satisfies $\hat{\theta}(\psi)(\hat{\theta}(\phi)(T))=0$ as well. From Definition 3.2. it is then immediate that $\operatorname{supp} \hat{\theta}(\phi)(T) \subset \operatorname{supp} T$.

To see that $\operatorname{supp} \hat{\theta}(\phi)(T) \subset \operatorname{supp} \phi$ as well, let $x \in G \backslash \operatorname{supp} \phi$, and assume towards a contradiction that $x \in \operatorname{supp} \hat{\theta}(\phi)(T)$. Since $x \notin \operatorname{supp} \phi$, there is a neighborhood $V$ of $x$ such that $\left.\phi\right|_{V} \equiv 0$. Take $\psi \in A(G)$ such that $\operatorname{supp} \psi \subseteq V$ and $\psi(x)=1$. Then $\psi \phi \equiv 0$ holds, so that $\hat{\theta}(\psi)(\hat{\theta}(\phi)(T))=\hat{\theta}(\psi \phi)(T)=0$. Since $x \in \operatorname{supp} \hat{\theta}(\phi)(T)$, this implies that $\psi(x)=0$, which is a contradiction.

The following is an alternative description of the support of an operator:

Proposition 3.3. Let $G$ be a locally compact group, and let $T \in \mathcal{B}\left(L^{2}(G)\right)$. Then

$$
\{\phi \in A(G): \hat{\theta}(\phi)(T)=0\}
$$

is an ideal of $A(G)$ whose hull equals supp $T$.

Proof. Let the ideal (2) be denoted by $I$. Then we have for $x \in G$ that

$$
\begin{aligned}
x \notin \operatorname{supp} T & \Longleftrightarrow \quad \text { there is } \phi \in A(G) \text { such that } \phi(x) \neq 0 \text { and } \hat{\theta}(\phi)(T)=0 \\
& \Longleftrightarrow \quad \text { there is } \phi \in I \text { with } \phi(x) \neq 0 \\
& \Longleftrightarrow x \notin \operatorname{hull}(I) .
\end{aligned}
$$

This completes the proof. 
Since $A(G)$ is Tauberian for any locally compact group $G$, the following is clear:

Corollary 3.4. Let $G$ be a locally compact group, and let $T \in \mathcal{B}\left(L^{2}(G)\right)$ be such that $\operatorname{supp} T=\varnothing$. Then $\hat{\theta}(\phi)(T)=0$ holds for all $\phi \in A(G)$.

Recall $([\mathrm{H}-\mathrm{K}])$ that a locally compact group $G$ is said to have the approximation property if the constant function 1 lies in the $w^{*}$-closure of $A(G)$ in $\mathcal{M}_{\mathrm{cb}}(A(G))$. Clearly, every amenable, locally compact group has the approximation property (by Leptin's theorem), but so does also every weakly amenable group in the sense of $\left[\mathrm{dC}-\mathrm{H}\right.$, such as $\mathbb{F}_{2}$, the free group in two generators. Nevertheless, the approximation property is weaker than weak amenability: by $\left[\underline{\mathrm{H}-\mathrm{K}}\right.$. Corollary 1.17 and Remark 3.10], the group $\mathbb{Z}^{2} \rtimes \mathrm{SL}(2, \mathbb{Z})$ has the approximation property, but is not weakly amenable.

Proposition 3.5. Let $G$ be a locally compact group with the approximation property. Then the following are equivalent for $T \in \mathcal{B}\left(L^{2}(G)\right)$ :

(i) $\operatorname{supp} T=\varnothing$;

(ii) $T=0$.

Proof. Of course, only (i) $\Longrightarrow$ (ii) needs proof.

Let $\left(e_{\alpha}\right)_{\alpha}$ be a net in $A(G)$ that converges to 1 in the $w^{*}$-topology of $\mathcal{M}_{\mathrm{cb}}(A(G))$. Since $\hat{\theta}$ is unital and $w^{*}-w^{*}$-continuous $(\underline{\mathbb{N}-\mathrm{R}-\mathrm{S}}$, Theorem 4.5$\left.]\right)$, it follows that $\operatorname{id}_{\mathcal{B}\left(L^{2}(G)\right)}=$ $w^{*}-\lim _{\alpha} \hat{\theta}\left(e_{\alpha}\right)$ and thus, by Corollary 3.4

$$
T=\hat{\theta}(1)(T)=w^{*}-\lim _{\alpha} \hat{\theta}\left(e_{\alpha}\right)(T)=0 .
$$

This proves (ii).

Remark. It is well possible that Proposition 3.5 is true for every locally compact group.

Let $G$ be a locally compact group, and let $F \subset G$ be closed. As is customary, we write

$$
I(F):=\left\{\phi \in A(G):\left.\phi\right|_{F} \equiv 0\right\} .
$$

We also define

$$
\mathcal{B}_{F}\left(L^{2}(G)\right):=\left\{T \in \mathcal{B}\left(L^{2}(G)\right): \operatorname{supp} T \subset F\right\} .
$$

Lemma 3.6. Let $G$ be a locally compact group, and let $F \subset G$ be a set of synthesis for $A(G)$. Then $\hat{\theta}(\phi)(T)=0$ holds for all $\phi \in I(F)$ and $T \in \mathcal{B}_{F}\left(L^{2}(G)\right)$.

Proof. Let $T \in \mathcal{B}_{F}\left(L^{2}(G)\right)$, and set

$$
I:=\{\phi \in A(G): \hat{\theta}(\phi)(T)=0\} .
$$

By Proposition 3.3 we have

$$
\operatorname{hull}(I)=\operatorname{supp} T \subset F .
$$

Since $F$ is a set of synthesis, this means that $I(F) \subset I$. 
Since the multiplication of $\mathcal{M}_{\mathrm{cb}}(A(G))$ is separately $w^{*}$-continuous (see [F-R-S], for instance), we obtain immediately (from the $w^{*}-w^{*}$-continuity of $\hat{\theta}$ ):

Corollary 3.7. Let $G$ be a locally compact group with the approximation property, let $F \subset G$ be a set of synthesis, and let $T \in \mathcal{B}_{F}\left(L^{2}(G)\right)$. Then $\hat{\theta}(\phi)(T)=0$ for all $\phi \in$ $\mathcal{M}_{\mathrm{cb}}(A(G))$ with $\left.\phi\right|_{F} \equiv 0$.

Proof. Let $\phi \in \mathcal{M}_{\mathrm{cb}}(A(G))$ be such that $\left.\phi\right|_{F} \equiv 0$, and let $\left(e_{\alpha}\right)_{\alpha}$ be a net in $A(G)$ converging to 1 in the $w^{*}$-topology of $\mathcal{M}_{\mathrm{cb}}(A(G))$. Then $\left(e_{\alpha} \phi\right)_{\alpha}$ is a net in $I(F)$ that is $w^{*}$-convergent to $\phi$. Lemma 3.6 and the $w^{*}-w^{*}$-continuity of $\hat{\theta}$ then yield the claim.

Let $G$ be a locally compact group, let $F \subset G$ be a set of synthesis, and let $T \in$ $\mathcal{B}_{F}\left(L^{2}(G)\right)$. Then it is clear from Lemma [3.6] that $\hat{\theta}(\phi)(T)$ for $\phi \in A(G)$ depends only on $\left.\phi\right|_{F}$ (if $G$ has the approximation property, this is even true for all $\phi \in \mathcal{M}_{\mathrm{cb}}(A(G)$ ) by Corollary [3.7).

Proposition 3.8. Let $G$ be a locally compact group, let $H$ be a closed subgroup of $G$, and let $T \in \mathcal{B}_{H}\left(L^{2}(G)\right)$. Then:

(i) if there is $\sigma \in A(G)$ with $\left.\sigma\right|_{H}=1$ and $\hat{\theta}(\sigma)(T)=T$, then $\hat{\theta}(\phi)(T)=\phi(e) T$ for all $\phi \in A(G)$ which are constant on $H$;

(ii) if $G$ has the approximation property, then $\hat{\theta}(\phi)(T)=\phi(e) T$ holds for all $\phi \in$ $\mathcal{M}_{\mathrm{cb}}(A(G))$ which are constant on $H$.

Proof. First, recall that $H$, as a closed subgroup, is a set of synthesis for $G$. ([T-T 2, Theorem 3]).

Suppose that there is $\sigma \in A(G)$ with $\left.\sigma\right|_{H}=1$ and $\hat{\theta}(\sigma)(T)=T$. Let $\phi \in A(G)$ be constant on $H$. Then $\phi(e) \sigma-\phi$ vanishes on $H$, so that, by Lemma 3.6 we have,

$$
0=\hat{\theta}(\phi(e) \sigma-\phi)(T)=\phi(e) \hat{\theta}(\sigma)(T)-\hat{\theta}(\phi)(T)=\phi(e) T-\hat{\theta}(\phi)(T) .
$$

This proves (i).

For (ii), just note that, if $\phi \in \mathcal{M}_{\mathrm{cb}}(A(G))$ is constant on $H$, then $\phi(e)-\phi$ vanishes on $H$. An application of Corollary 3.7 then yields (ii).

As we already noted, our notion of support coincides with the one from Eym for operators in $\mathrm{VN}(G)$. We now compute the support of multiplication operators:

Example. Let $G$ be a locally compact group, and let $f \in L^{\infty}(G)$. For any $\phi \in A(G)$, we have

$$
\hat{\theta}(\phi)\left(M_{f}\right)=M_{f} \hat{\theta}(\phi)(1)=\phi(e) M_{f},
$$

where the first equality is due to the fact that $\hat{\theta}(\phi)$ is an $L^{\infty}(G)$-bimodule homomorphism. If $x \in G \backslash\{e\}$, we can find $\phi \in A(G)$ with $\phi(x) \neq 0=\phi(e)$, so that $\hat{\theta}(\phi)\left(M_{f}\right)=0$ by 
(3). Hence, $x$ cannot lie in $\operatorname{supp} M_{f}$. Since $x \in G \backslash\{e\}$ was arbitrary, this means that $M_{f} \in \mathcal{B}_{e}\left(L^{2}(G)\right)$. Moreover, it is also clear from (3) that $e \in \operatorname{supp} M_{f}$ whenever $f \neq 0$, i.e., $\operatorname{supp} M_{f}=\{e\}$.

\section{Harmonic operators: the dual picture}

Let $G$ be a locally compact group, and let $\theta: M(G) \rightarrow \mathcal{C B}\left(\mathcal{B}\left(L^{2}(G)\right)\right)$ be the completely isometric representation from Gha] (see also [Neu 1], Neu 2], and-for abelian $G$ - St $\varnothing$ ) given by

$$
\langle\theta(\mu) T, \omega\rangle:=\int_{G}\left\langle\rho(t) T \rho\left(t^{-1}\right), \omega\right\rangle d \mu(t) \quad\left(\mu \in M(G), T \in \mathcal{B}\left(L^{2}(G)\right), \omega \in \mathcal{T}\left(L^{2}(G)\right)\right) .
$$

For $\mu \in M^{1}(G)$, W. Jaworski and the first-named author define $T \in \mathcal{B}\left(L^{2}(G)\right)$ to be $\mu$-harmonic if $\theta(\mu)(T)=T(\underline{\mathrm{J}-\mathrm{N}]})$. Since

$$
\theta(\mu) M_{\phi}=M_{\mu * \phi} \quad\left(\phi \in L^{\infty}(G)\right),
$$

this generalizes the notion of a $\mu$-harmonic function. More precisely, denoting the space of $\mu$-harmonic functions and operators by $\mathcal{H}_{\mu}$ and $\tilde{\mathcal{H}}_{\mu}$, respectively, this shows that $\mathcal{H}_{\mu} \subseteq \tilde{\mathcal{H}}_{\mu}$. Moreover, $\tilde{\mathcal{H}}_{\mu}$ carries a natural multiplication extending the one of $\mathcal{H}_{\mu}$-and turning $\tilde{\mathcal{H}}_{\mu}$ into a non-commutative von Neumann algebra $([\underline{\mathrm{J}-\mathrm{N}}])$ : given $S, T \in \tilde{\mathcal{H}}_{\mu}$, their product in $\tilde{\mathcal{H}}_{\mu}$ is explicitly given by

$$
w^{*}-\lim _{n} \int_{G} \rho(x)(S T) \rho\left(x^{-1}\right) \mu^{* n}(x) .
$$

One of the main results obtained in $[\mathrm{J}-\mathrm{N}]$ - affirmatively answering a question by $\mathrm{M}$. Izumi (【zu $]$ — consists of a precise structural description of $\tilde{\mathcal{H}}_{\mu}$ : provided that $G$ is second countable, $\tilde{\mathcal{H}}_{\mu}$ is exactly the crossed product of $\mathcal{H}_{\mu}$ with $G$ acting by left translation ([J-N] Theorem 6.3]). In particular, it shows that the algebra $\operatorname{VN}(G)$, as a subalgebra of $\tilde{\mathcal{H}}_{\mu}$, plays the same rôle as the scalars for the classical algebra of harmonic functions $\mathcal{H}_{\mu}$.

Using $\hat{\theta}: \mathcal{M}_{\mathrm{cb}}(A(G)) \rightarrow \mathcal{C B}\left(\mathcal{B}\left(L^{2}(G)\right)\right)$ from $[\mathrm{N}-\mathrm{R}-\mathrm{S}]$, we can extend the notion of a $\sigma$-harmonic functional on $A(G)$ from $\mathrm{C}-\mathrm{L}$ to that of a $\sigma$-harmonic operator in a way analogous to the passage from $\mathcal{H}_{\mu}$ to $\tilde{\mathcal{H}}_{\mu}$ via $\theta$ :

Definition 4.1. Let $G$ be a locally compact group, and let $\sigma \in \mathcal{M}_{\mathrm{cb}}(A(G))$. Then an operator $T \in \mathcal{B}\left(L^{2}(G)\right)$ is called $\sigma$-harmonic if $\hat{\theta}(\sigma)(T)=T$. We denote the collection of all $\sigma$-harmonic operators on $L^{2}(G)$ by $\tilde{\mathcal{H}}_{\sigma}$.

Remarks. 1. Obviously, $\tilde{\mathcal{H}}_{\sigma}$ is a $w^{*}$-closed subspace of $\mathcal{B}\left(L^{2}(G)\right)$.

2. Trivially, $\tilde{\mathcal{H}}_{\sigma}$ contains $\mathcal{H}_{\sigma}$, and since,

$$
\hat{\theta}(\phi)\left(M_{f}\right)=M_{f} \hat{\theta}(\phi)(1)=M_{f} \quad\left(f \in L^{\infty}(G)\right),
$$

it contains $L^{\infty}(G)$ as well. 
As proven in $\mathrm{C}-\mathrm{L}], \mathcal{H}_{\sigma}$ is a von Neumann subalgebra of $\operatorname{VN}(G)$ for $\sigma \in P^{1}(G)$, which stands in marked contrast to $\mathcal{H}_{\mu}$ with $\mu \in M^{1}(G)$. In the remainder of this section, we shall see that a similar statement is true for $\tilde{\mathcal{H}}_{\sigma}$ : under some, fairly mild, additional hypotheses, it is a von Neumann subalgebra of $\mathcal{B}\left(L^{2}(G)\right)$; in fact, we shall prove that

$$
\tilde{\mathcal{H}}_{\sigma}=\left(\mathcal{H}_{\sigma} \cup L^{\infty}(G)\right)^{\prime \prime}
$$

Remark. The description (4) may be viewed as a dual version of the central structural result [J-N] Theorem 6.3]; in our setting, it is the algebra $L^{\infty}(G)$ which plays the rôle of the scalars. Noting that $L^{\infty}(G)$ and $\operatorname{VN}(G)$ are Kac algebras dual to each other (see $[\mathrm{E}-\mathrm{S}]$ ), we are inclined to believe that one may find one single structure result that unifies those descriptions of $\tilde{\mathcal{H}}_{\sigma}$ and $\tilde{\mathcal{H}}_{\mu}$ in the general framework of Kac algebras (or, even more generally, locally compact quantum groups).

We proceed through a series of lemmas and propositions.

Proposition 4.2. Let $G$ be a locally compact group, and let $\sigma \in P^{1}(G)$. Then

$$
\left(\mathcal{H}_{\sigma} \cup L^{\infty}(G)\right)^{\prime \prime} \subset \tilde{\mathcal{H}}_{\sigma}
$$

holds.

Proof. By von Neumann's double commutant theorem, $\left(\mathcal{H}_{\sigma} \cup L^{\infty}(G)\right)^{\prime \prime}$ is the von Neumann subalgebra of $\mathcal{B}\left(L^{2}(G)\right)$ generated by $\mathcal{H}_{\sigma}$ and $L^{\infty}(G)$. Since $\tilde{\mathcal{H}}_{\sigma}$ is a $w^{*}$-closed subspace of $\mathcal{B}\left(L^{2}(G)\right)$, it is sufficient to show that finite products of operators from $\mathcal{H}_{\sigma} \cup L^{\infty}(G)$ belong to $\tilde{\mathcal{H}}_{\sigma}$. Moreover, since $\mathcal{H}_{\sigma} \cong \mathrm{VN}\left(G_{\sigma}\right)$, it is enough to consider products of the form

$$
\prod_{j=1}^{n} \lambda\left(x_{j}\right) M_{f_{j}},
$$

where $x_{1}, \ldots, x_{n} \in G_{\sigma}$ and $f_{1}, \ldots, f_{n} \in L^{\infty}(G)$. Since

$$
\lambda(x) M_{f}=M_{\lambda(x) f} \lambda(x) \quad\left(x \in G, f \in L^{\infty}(G)\right),
$$

a simple induction on the number of factors in (5) shows that (15) is, in fact, of the form $M_{f} \lambda(x)$ with $f \in L^{\infty}(G)$ and $x \in G_{\sigma}$. Since $\hat{\theta}(\sigma)$ is an $L^{\infty}(G)$-bimodule homomorphism and since $\hat{\theta}(\sigma)$ fixes $\lambda(x)$ if $x \in G_{\sigma}$, we have

$$
\hat{\theta}(\sigma)\left(M_{f} \lambda(x)\right)=M_{f} \hat{\theta}(\sigma)(\lambda(x))=M_{f} \lambda(x) \quad\left(f \in L^{\infty}(G), x \in G_{\sigma}\right) .
$$

i.e., $M_{f} \lambda(x) \in \tilde{\mathcal{H}}_{\sigma}$.

In view of the foregoing remarks, this completes the proof. 
While Proposition 4.2 provides an estimate for $\tilde{\mathcal{H}}_{\sigma}$ "from below", we now give one "from above".

Extending our previous notation, we set $G_{\sigma}:=\{x \in G: \sigma(x)=1\}$ for any $\sigma \in$ $\mathcal{M}_{\mathrm{cb}}(A(G))$. Note that $G_{\sigma}$ need not be a subgroup of $G$ unless $\sigma \in P^{1}(G)$, but that it is still a closed subset of $G$, so that the conclusion of the following proposition still makes sense.

Proposition 4.3. Let $G$ be a locally compact group, and let $\sigma \in \mathcal{M}_{\mathrm{cb}}(A(G))$. Then we have $\tilde{\mathcal{H}}_{\sigma} \subset \mathcal{B}_{G_{\sigma}}\left(L^{2}(G)\right)$.

Proof. Let $T \in \tilde{\mathcal{H}}_{\sigma}$, and let $x \in G \backslash G_{\sigma}$, i.e., $\sigma(x) \neq 1$. Let $\phi \in A(G)$ be such that $\phi(x) \neq 0$, so that $\psi(x):=\phi(x) \sigma(x)-\phi(x) \neq 0$. On the other hand, we have

$$
\hat{\theta}(\psi)(T)=\hat{\theta}(\phi \sigma)(T)-\hat{\theta}(\phi)(T)=\hat{\theta}(\phi)(\hat{\theta}(\sigma)(T))-\hat{\theta}(\phi)(T)=\hat{\theta}(\phi)(T)-\hat{\theta}(\phi)(T)=0 .
$$

This means that $x \notin \operatorname{supp} T$.

Let $G$ be a locally compact group, and let $H$ be a closed subgroup. We define

$$
L^{\infty}(G: H):=\left\{\phi \in L^{\infty}(G): \lambda(x) \phi=\phi \text { for all } x \in H\right\} .
$$

It is obvious that $L^{\infty}(G: H)$ is $w^{*}$-closed in $L^{\infty}(G)$. Slightly deviating from [For], we set

$$
B(G: H):=\{\phi \in B(G): \lambda(x) \phi=\phi \text { for all } x \in H\} .
$$

(Note that in For], right instead of left translates are considered.) We can canonically embed $B(G: H)$ into $L^{\infty}(G: H)$. Also, we set

$$
P_{H}^{1}(G):=\left\{\sigma \in P^{1}(G):\left.\sigma\right|_{H} \equiv 1\right\}
$$

As is observed in $\left[\mathrm{K}-\mathrm{L}\right.$, the functions in $P_{H}^{1}(G)$ are constant on both left and right cosets of $H$ and thus are contained in $B(G: H)$. Following $[\mathrm{K}-\mathrm{L}$, we say that $G$ has the $H$ separation property if, for each $x \in G \backslash H$, there is $\sigma \in P_{H}^{1}(G)$ such that $\sigma(x) \neq 1$. For instance, whenever $H$ is open, compact, or neutral - this includes all normal subgroups as well as all closed subgroups of [SIN]-groups-, $G$ has the $H$-separation property (see $[\mathrm{K}-\mathrm{L}]$.

Lemma 4.4. Let $G$ be a locally compact group, and let $H$ be a closed subgroup of $G$ such that $G$ has the $H$-separation property. Then $B(G: H)$ is $w^{*}$-dense in $L^{\infty}(G: H)$.

Proof. Let $\mathfrak{M}$ denote the $w^{*}$-closure of the linear span of all right translates of all functions in $P_{H}^{1}(G)$ in $L^{\infty}(G)$. Then $\mathfrak{M}$ is a von Neumann subalgebra of $L^{\infty}(G)$ and invariant under right translation. Set

$$
L:=\{x \in G: \lambda(x) \phi=\phi \text { for all } \phi \in \mathfrak{M}\}
$$


Then $L$ is a closed subgroup of $G$ containing $H$, and from [T-T 1, Theorem 2] (see also [Lau, Lemma 3.2]), we conclude that $\mathfrak{M}=L^{\infty}(G: L)$.

Assume that there is $x \in L \backslash H$. Since $G$ has the $H$-separation property, there is $\sigma \in P_{H}^{1}(G) \subset L^{\infty}(G: L)$ such that $\sigma(x) \neq 1$ and thus $\lambda\left(x^{-1}\right) \sigma \neq \sigma$. This is a contradiction, so that $H=L$.

For any locally compact group $G$ and $\sigma \in P^{1}(G)$, it is clear by definition that $G$ has the $G_{\sigma}$-separation property. Hence, we obtain:

Corollary 4.5. Let $G$ be a locally compact group, and let $\sigma \in P^{1}(G)$. Then $B\left(G: G_{\sigma}\right)$ is $w^{*}$-dense in $L^{\infty}\left(G: G_{\sigma}\right)$.

Lemma 4.6. Let $G$ be a locally compact group, let $H$ be a closed subgroup, and let $\phi \in$ $B(G: H)$. Then there are a unitary representation $\pi$ of $G$ on a Hilbert space $\mathfrak{H}$ as well as $\xi, \eta \in \mathfrak{H}$ such that

$$
\phi(x)=\langle\pi(x) \xi, \eta\rangle \quad(x \in G)
$$

and $\pi(y) \eta=\eta$ for all $y \in H$.

Proof. By definition of $B(G)$, there are a unitary representation $\pi$ of $G$ on a Hilbert space $\mathfrak{H}$ as well as $\xi, \eta_{0} \in \mathfrak{H}$ such that

$$
\phi_{\xi, \eta_{0}}(x):=\left\langle\pi(x) \xi, \eta_{0}\right\rangle=\phi(x) \quad(x \in G) .
$$

Without loss of generality, suppose that $\|\xi\|=1$. Set

$$
C:=\left\{\eta \in \mathfrak{H}:\|\eta\| \leq\left\|\eta_{0}\right\| \text { and } \phi_{\xi, \eta}=\phi\right\}
$$

Then $C$ is a non-empty, convex, weakly compact subset of $\mathfrak{H}$. Let $\eta \in C$ and $y \in H$. Then we have

$$
\phi_{\xi, \pi(y) \eta}(x)=\langle\pi(x) \xi, \pi(y) \eta\rangle=\left\langle\pi\left(y^{-1} x\right) \xi, \eta\right\rangle=(\lambda(y) \phi)(x)=\phi(x) \quad(x \in G),
$$

so that $\pi(y) \eta \in C$ again. From the Ryll-Nardzewski fixed point theorem ( $\mathrm{G}-\mathrm{D}$, (9.6) Theorem]), we conclude that there is $\eta \in C$ with $\pi(y) \eta=\eta$ for $y \in H$.

Proposition 4.7. Let $G$ be a locally compact group, let $H$ be a closed subgroup of $G$, let $T \in \mathcal{B}_{H}\left(L^{2}(G)\right)$, and suppose that one of the following holds:

(a) there is $\sigma \in A(G)$ with $\left.\sigma\right|_{H}=1$ and $\hat{\theta}(\sigma)(T)=T$;

(b) $G$ has the approximation property and the $H$-separation property.

Then $T$ lies in $\left(\mathrm{VN}(H) \cup L^{\infty}(G)\right)^{\prime \prime}$. 
For the proof, we first establish some conventions for (possibly infinite) matrices. The extensive use of matrix calculations may seem like an unnecessary complication at the first glance, but appears to be unavoidable in view of how $\hat{\theta}: \mathcal{M}_{\mathrm{cb}}(A(G)) \rightarrow \mathcal{C B}\left(\mathcal{B}\left(L^{2}(G)\right)\right.$ is defined in $[\mathrm{N}-\mathrm{R}-\mathrm{S}$, namely via the extended Haagerup tensor product of operator spaces, an object that itself is defined in terms of arbitrarily large matrices.

Given a linear space $E$ and index sets $\mathbb{I}$ and $\mathbb{J}$, we denote by $M_{\mathbb{I} \times \mathbb{J}}(E)$ the matrices $\left[x_{i, j}\right]_{\substack{i \in \mathbb{I} \\ j \in \mathbb{J}}}$ with $x_{i, j} \in E$ for $(i, j) \in \mathbb{I} \times \mathbb{J}$. If $\mathbb{I}=\mathbb{J}$, we write $M_{\mathbb{J}}(E)$, and if $E=\mathbb{C}$, we simply use the symbols $M_{\mathbb{I} \times \mathbb{J}}$ and $M_{\mathbb{J}}$ instead of $M_{\mathbb{I} \times \mathbb{J}}(\mathbb{C})$ and $M_{\mathbb{J}}(\mathbb{C})$, respectively. For $x \in E$ and $\left[\alpha_{i, j}\right]_{\substack{i \in \mathbb{I} \\ j \in \mathbb{J}}}$, we set $\left[\alpha_{i, j}\right]_{\substack{i \in \mathbb{I} \\ j \in \mathbb{J}}} \otimes x=\left[\alpha_{i, j} x\right]_{\substack{i \in \mathbb{I} \\ j \in \mathbb{J}}}$. We convene to interpret families $\left[x_{j}\right]_{j \in \mathbb{J}}$ as row vectors, and write $\left[x_{j}\right]_{j \in \mathbb{J}}^{t}$ for the corresponding column vector. Finally, we denote by $1_{\mathbb{J}}$ the matrix $\left[\delta_{j, k}\right]_{j, k \in \mathbb{J}}$, and set $0_{\mathbb{I} \times \mathbb{J}}=\left[\alpha_{i, j}\right]_{\substack{i \in \mathbb{I} \\ j \in \mathbb{J}}}$ with $\alpha_{i, j}=0$ for $(i, j) \in \mathbb{I} \times \mathbb{J}$.

Proof. If (a) holds, $H$ is necessarily compact, so that in both case (a) and case (b), $G$ has the $H$-separation property. By Lemma 4.4 $B(G: H)$ is therefore $w^{*}$-dense in $L^{\infty}(G: H)$. We will show that $M_{\phi} T=T M_{\phi}$ for all $\phi \in B(G: H)$, so that $T$ lies in $L^{\infty}(G: H)^{\prime}$. Since, as is easily checked,

$$
\left(\mathrm{VN}(H) \cup L^{\infty}(G)\right)^{\prime}=L^{\infty}(G: H)
$$

this will prove the proposition.

We adapt part of the proof of $[\mathrm{N}-\mathrm{R}-\mathrm{S}$, Theorem 5.1] to our situation.

Let $\phi \in B(G: H)$. By Lemma 4.6, there thus are a unitary representation $\pi$ of $G$ on some Hilbert space $\mathfrak{H}$ as well as $\xi, \eta \in \mathfrak{H}$ such that

$$
\phi(x)=\langle\pi(x) \xi, \eta\rangle \quad(x \in G)
$$

and $\pi(y) \eta=\eta$ for $y \in H$. Let

$$
\mathfrak{K}:=\{\zeta \in \mathfrak{H}: \pi(y) \zeta=\zeta \text { for } y \in H\},
$$

so that, in particular, $\eta \in \mathfrak{K}$. Let $\left(e_{i}\right)_{i \in \mathbb{I}}$ be an orthonormal basis for $\mathfrak{K}$, and extend it to an orthonormal basis $\left(e_{i}\right)_{i \in \mathbb{J}}$ for $\mathfrak{H}$ (so that, in particular, $\mathbb{I} \subset \mathbb{J}$ ). For $(i, k) \in \mathbb{J} \times \mathbb{J}$ set

$$
\phi_{i, k}(x):=\left\langle\pi(x) e_{k}, e_{i}\right\rangle \quad \text { and } \quad \check{\phi}_{i, k}(x):=\phi_{i, k}\left(x^{-1}\right) \quad(x \in G)
$$

Since $e_{i} \in \mathfrak{K}$, it follows that $\phi_{i, k} \in B(G: H)$ for all $(i, k) \in \mathbb{I} \times \mathbb{J}$. By the definition of $\hat{\theta}$, we have

$$
\hat{\theta}\left(\phi_{i, k}\right)(T)=\sum_{j \in \mathbb{J}} M_{\phi_{i, j}} T M_{\check{\phi}_{j, k}} \quad((i, k) \in \mathbb{I} \times \mathbb{J}) ;
$$

By Proposition 3.8(i) or (ii) - depending on whether (a) or (b) is satisfied-, the left hand side of (6) equals $\phi_{i, k}(e) T$ for all $(i, k) \in \mathbb{I} \times \mathbb{J}$, so that (6) becomes

$$
\phi_{i, k}(e) T=\sum_{j \in \mathbb{J}} M_{\phi_{i, j}} T M_{\check{\phi}_{j, k}} \quad((i, k) \in \mathbb{I} \times \mathbb{J}) .
$$


Interpreting (7]) as a matrix identity — with matrices indexed over $\mathbb{J} \times \mathbb{J}$ 一, we obtain

$$
\phi_{i, k}(e) T=\left[\phi_{i, j}\right]_{j \in \mathbb{J}}\left(1_{\mathbb{J}} \otimes T\right)\left[\check{\phi}_{j, k}\right]_{j \in \mathbb{J}} \quad((i, k) \in \mathbb{I} \times \mathbb{J}) .
$$

We view $\Phi:=\left[\phi_{i, k}\right]_{i, k \in \mathbb{J}}$ as an element of $M_{\mathbb{J}}\left(\mathcal{C}_{b}(G)\right)$ and set $\check{\Phi}:=\left[\check{\phi}_{i, k}\right]_{i, k \in \mathbb{J}}$. Then $\check{\Phi}$ also lies in $M_{\mathbb{J}}\left(\mathcal{C}_{b}(G)\right)$ and satisfies

$$
\Phi \check{\Phi}=1_{\mathbb{J}} \otimes 1=\check{\Phi} \Phi .
$$

Furthermore, set $\Psi:=\left[\phi_{i, k}\right]_{\substack{i \in \mathbb{I} \\ k \in \mathbb{J}}} \in M_{\mathbb{I} \times \mathbb{J}}\left(\mathcal{C}_{b}(G)\right)$. Since $\phi_{i, k}(e)=\delta_{i, k}$ for $(i, k) \in \mathbb{I} \times \mathbb{J}$, we obtain from (8) that

$$
\begin{aligned}
\left(1_{\mathbb{I}} \oplus 0_{\mathbb{I} \times(\mathbb{I} \backslash \mathbb{I})}\right) \otimes T & =\left[\phi_{i, k}(e) T\right]_{\substack{i \in \mathbb{I} \\
k \in \mathbb{J}}} \\
& =\left[\left[\phi_{i, j}\right]_{j \in \mathbb{J}}\left(1_{\mathbb{J}} \otimes T\right)\left[\check{\phi}_{j, k}\right]_{j \in \mathbb{J}}\right]_{\substack{i \in \mathbb{I} \\
k \in \mathbb{J}}} \\
& =\Psi\left(1_{\mathbb{J}} \otimes T\right) \check{\Phi},
\end{aligned}
$$

and thus, by (9)

$$
\Psi\left(1_{\mathbb{J}} \otimes T\right)=\left(\left(1_{\mathbb{I}} \oplus 0_{\mathbb{I} \times(\mathbb{I} \backslash \mathbb{I})}\right) \otimes T\right) \Phi .
$$

Let $\left[\alpha_{j}\right]_{j \in \mathbb{J}}$ and $\left[\beta_{i}\right]_{i \in \mathbb{I}}$ be in $\ell^{2}(\mathbb{J})$ and $\ell^{2}(\mathbb{I})$, respectively, such that $\xi=\sum_{j \in \mathbb{J}} \alpha_{j} e_{j}$ and $\eta=\sum_{i \in \mathbb{I}} \beta_{i} e_{i}$; it follows that

$$
\phi(x)=\langle\pi(x) \xi, \eta\rangle=\sum_{\substack{j \in \mathbb{J} \\ i \in \mathbb{I}}} \alpha_{j}\left\langle\pi(x) e_{j}, e_{i}\right\rangle \bar{\beta}_{i}=\sum_{\substack{j \in \mathbb{J} \\ i \in \mathbb{I}}} \alpha_{j} \phi_{i, j}(x) \bar{\beta}_{i} \quad(x \in G)
$$

or, in matrix notation,

$$
\phi=\left[\bar{\beta}_{i}\right]_{i \in \mathbb{I}}^{t} \Psi\left[\alpha_{j}\right]_{j \in \mathbb{J}} .
$$

Hence, we obtain eventually:

$$
\begin{aligned}
M_{\phi} T & =\left[\bar{\beta}_{i}\right]_{i \in \mathbb{I}}^{t} \Psi\left(1_{\mathbb{J}} \otimes T\right)\left[\alpha_{j}\right]_{j \in \mathbb{J}} \\
& =\left[\bar{\beta}_{i}\right]_{i \in \mathbb{I}}^{t}\left(\left(1_{\mathbb{I}} \oplus 0_{\mathbb{I} \times(\mathbb{I} \backslash \mathbb{I})}\right) \otimes T\right) \Phi\left[\alpha_{j}\right]_{j \in \mathbb{J}} \\
& =T M_{\phi} .
\end{aligned}
$$

This proves the claim.

We can now prove the main result of this section:

Theorem 4.8. Let $G$ be a locally compact group, and let $\sigma \in P^{1}(G)$. Then the following hold:

(i) if $\sigma \in A(G)$, then $\tilde{\mathcal{H}}_{\sigma}=\left(\mathcal{H}_{\sigma} \cup L^{\infty}(G)\right)^{\prime \prime}$;

(ii) if $G$ has the approximation property, then

$$
\tilde{\mathcal{H}}_{\sigma}=\left(\mathcal{H}_{\sigma} \cup L^{\infty}(G)\right)^{\prime \prime}=\mathcal{B}_{G_{\sigma}}\left(L^{2}(G)\right) .
$$


In either case, $\tilde{\mathcal{H}}_{\sigma}=\left(\mathcal{H}_{\sigma} \cup L^{\infty}(G)\right)^{\prime \prime}$ is a von Neumann subalgebra of $\mathcal{B}\left(L^{2}(G)\right)$.

Proof. By Propositions 4.2 and 4.3

$$
\left(\mathcal{H}_{\sigma} \cup L^{\infty}(G)\right)^{\prime \prime} \subset \tilde{\mathcal{H}}_{\sigma} \subset \mathcal{B}_{G_{\sigma}}\left(L^{2}(G)\right)
$$

holds without any additional hypotheses.

In case (i), we conclude from Proposition 4.7 (with condition (a)) that $\tilde{\mathcal{H}}_{\sigma} \subset\left(\mathcal{H}_{\sigma} \cup\right.$ $\left.L^{\infty}(G)\right)^{\prime \prime}$. For case (ii), recall that, as we remarked before Corollary 4.5, $G$ has the $G_{\sigma^{-}}$-separation property. Hence, by Proposition 4.7 (with condition (b)), we even have $\mathcal{B}_{G_{\sigma}}\left(L^{2}(G)\right) \subset\left(\mathcal{H}_{\sigma} \cup L^{\infty}(G)\right)^{\prime \prime}$. This proves the theorem.

Remark. We believe, but have been unable to prove, that

$$
\tilde{\mathcal{H}}_{\sigma}=\left(\mathcal{H}_{\sigma} \cup L^{\infty}(G)\right)^{\prime \prime}=\mathcal{B}_{G_{\sigma}}\left(L^{2}(G)\right)
$$

holds without any additional hypotheses on $\sigma$ or $G$.

For adapted $\sigma$, we obtain as a special case:

Corollary 4.9. Let $G$ be a locally compact group, and let $\sigma \in P^{1}(G)$ be adapted. Then the following hold:

(i) if $\sigma \in A(G)$, then $\tilde{\mathcal{H}}_{\sigma}=L^{\infty}(G)$;

(ii) if $G$ has the approximation property, then

$$
\tilde{\mathcal{H}}_{\sigma}=L^{\infty}(G)=\mathcal{B}_{e}\left(L^{2}(G)\right) .
$$

In either case, $\tilde{\mathcal{H}}_{\sigma}=L^{\infty}(G)$ is a von Neumann subalgebra of $\mathcal{B}\left(L^{2}(G)\right)$.

\section{Harmonic operators through ideals in the predual}

An alternative, very fruitful approach to the classical space $\mathcal{H}_{\mu}$ of $\mu$-harmonic functions (where $\mu$ is a probability measure) has been carried out by G. A. Willis (Wil]). There, the study of $\mathcal{H}_{\mu}$ is transferred to its pre-annihilator

$$
J_{\mu}:={\overline{\left\{f-f * \mu: f \in L^{1}(G)\right.}}^{L^{1}(G)}
$$

in $L^{1}(G)$, which (obviously) forms a left ideal in the group algebra. In order to investigate the non-commutative analogue $\tilde{\mathcal{H}}_{\mu}$ of $\mathcal{H}_{\mu}$ in a similar fashion, W. Jaworski and the first-named author use the "quantized" convolution product in the space $\mathcal{T}\left(L^{2}(G)\right)=$ $\mathcal{B}\left(L^{2}(G)\right)_{*}$ of trace class operators, as introduced and studied in Neu 1] and [Neu 3] (see also $\mathrm{Pir}$ for further results on this product). Indeed, they show that the pre-annihilator

$$
\tilde{J}_{\mu}:=\overline{\left\{\omega-\theta(\mu)_{*}(\omega): \omega \in \mathcal{T}\left(L^{2}(G)\right)\right\}} \mathcal{T}\left(L^{2}(G)\right)
$$


of $\tilde{\mathcal{H}}_{\mu}$ in $\mathcal{T}\left(L^{2}(G)\right)$ forms a left ideal with respect to this convolution ([J-N] Proposition 3.3]).

We shall first indicate how to equip $\mathcal{T}\left(L^{2}(G)\right)$ with a product that turns it into a completely contractive Banach algebra, and may be thought of as a "non-commutative pointwise product", in other words, a Fourier algebra type product, instead of a convolution type product, as described above. Just as the latter, the multiplication we shall consider here is also very different from the ordinary composition of operators. From a Hopf-von Neumann algebraic point of view, both the convolution type and the Fourier algebra type product on the space $\mathcal{T}\left(L^{2}(G)\right)$ are constructed in exactly the same way: the first one is derived from a co-multiplication on $\mathcal{B}\left(L^{2}(G)\right)$ that canonically extends the one of $L^{\infty}(G)$ whereas the second one is based on a canonical extension of the comultiplication of $\operatorname{VN}(G)$. In this sense, both products on $\mathcal{T}\left(L^{2}(G)\right)$ are "dual" to each other.

Let $G$ be a locally compact group. We define a unitary operator $W \in \mathcal{B}\left(L^{2}(G \times G)\right)$ by letting

$$
(W \xi)(x, y):=\xi(x, x y) \quad\left(\xi \in L^{2}(G), x, y \in G\right) .
$$

Identifying $L^{2}(G \times G)$ with $L^{2}(G) \tilde{\otimes}_{2} L^{2}(G)$ (Hilbert space tensor product), we denote the flip map on $L^{2}(G \times G)$ by $\sigma$. Then $\hat{W}:=\sigma W^{*} \sigma$ is a again unitary, and

$$
\hat{\Gamma}: \mathcal{B}\left(L^{2}(G)\right) \rightarrow \mathcal{B}\left(L^{2}(G \times G)\right), \quad T \mapsto \hat{W}(1 \otimes T) \hat{W}^{*}
$$

is a co-multiplication, i.e., a normal, unital, injective ${ }^{*}$-homomorphism satisfying

$$
(\hat{\Gamma} \otimes \mathrm{id}) \circ \hat{\Gamma}=(\mathrm{id} \otimes \hat{\Gamma}) \circ \hat{\Gamma}
$$

The pre-adjoint

$$
\hat{\Gamma}: \mathcal{T}\left(L^{2}(G)\right) \hat{\otimes} \mathcal{T}\left(L^{2}(G)\right) \rightarrow \mathcal{T}\left(L^{2}(G)\right)
$$

where $\hat{\otimes}$ denotes the projective tensor product of operator spaces, is a complete contraction and turns $\mathcal{T}\left(L^{2}(G)\right)$ into a completely contractive Banach algebra. In order to tell this product on $\mathcal{T}\left(L^{2}(G)\right)$ apart from the usual composition of operators, we denote it by $\bullet$, so that

$$
\langle\omega \bullet \rho, T\rangle:=\langle\omega \otimes \rho, \hat{\Gamma}(T)\rangle \quad\left(\omega, \rho \in \mathcal{T}\left(L^{2}(G)\right), T \in \mathcal{B}\left(L^{2}(G)\right)\right) .
$$

Furthermore, the pre-adjoint of the inclusion $\operatorname{VN}(G) \subset \mathcal{B}\left(L^{2}(G)\right)$ is an algebra homomorphism $\pi: \mathcal{T}\left(L^{2}(G)\right) \rightarrow A(G)$ (and necessarily a complete quotient map), and $\mathcal{B}\left(L^{2}(G)\right.$ ) becomes a completely contractive $\mathcal{T}\left(L^{2}(G)\right)$-bimodule through

$$
\omega \cdot T:=(\operatorname{id} \otimes \omega)(\hat{\Gamma}(T)) \quad \text { and } \quad T \cdot \omega:=(\omega \otimes \mathrm{id})(\hat{\Gamma}(T))
$$

$$
\left(\omega \in \mathcal{T}\left(L^{2}(G)\right), T \in \mathcal{B}\left(L^{2}(G)\right)\right) .
$$


(These module actions have been studied in $[\mathrm{N}-\mathrm{R}-\mathrm{S}]$ and $[\mathrm{P}-\mathrm{R}]$.)

Since $\hat{W} \in \operatorname{VN}(G) \bar{\otimes} L^{\infty}(G)$, we have

$$
\omega \cdot T=(\mathrm{id} \otimes \omega)\left(\hat{W}(1 \otimes T) \hat{W}^{*}\right) \in \mathrm{VN}(G) \quad\left(\omega \in \mathcal{T}\left(L^{2}(G)\right), T \in \mathcal{B}\left(L^{2}(G)\right)\right) .
$$

Hence, the module action induces a complete contraction $\hat{\mathcal{S}}: \mathcal{T}\left(L^{2}(G)\right) \hat{\otimes} \mathcal{B}\left(L^{2}(G)\right) \rightarrow$ $\operatorname{VN}(G)$. In $[\mathrm{P}-\mathrm{R}, \operatorname{UCQ}(G)$ is defined to be the range of $\hat{\mathcal{S}}$ in $\operatorname{VN}(G)$, equipped with the quotient operator space structure. The dual space $\operatorname{UCQ}(G)^{*}$ then naturally becomes a completely contractive Banach algebra, which contains $\mathcal{M}_{\mathrm{cb}} A(G)$ as a closed subalgebra, and the adjoint $\hat{\mathcal{S}}^{*}$ extends the representation $\hat{\theta}$ from $\mathcal{M}_{\mathrm{cb}}(A(G))$ to $\operatorname{UCQ}(G)^{*}$. More precisely, if, for $n \in \operatorname{UCQ}(G)^{*}$ and $T \in \mathcal{B}\left(L^{2}(G)\right)$, one defines $n \diamond T \in \mathcal{B}\left(L^{2}(G)\right)$ through

$$
\langle n \diamond T, \omega\rangle:=\langle n, \omega \cdot T\rangle \quad\left(\omega \in \mathcal{T}\left(L^{2}(G)\right)\right)
$$

then, for each $n \in \operatorname{UCQ}(G)^{*}$, the map

$$
\mathcal{B}\left(L^{2}(G)\right) \rightarrow \mathcal{B}\left(L^{2}(G)\right), \quad T \mapsto n \diamond T
$$

is a completely bounded operator on $\mathcal{B}\left(L^{2}(G)\right)$, which we denote by $\tilde{\hat{\theta}}(n)$. It is easy to check that $\tilde{\hat{\theta}}=\hat{\mathcal{S}}^{*}$. For more information, see [P-R] (and [N-R-S] for the amenable case).

In the sequel, we shall use the fact that $\hat{\theta}$ and $\hat{\theta}$ coincide on $A(G)$, which follows from $[\mathrm{P}-\mathrm{R}]$ (or $[\mathrm{N}-\mathrm{R}-\mathrm{S}]$ if $G$ is amenable). For the reader's convenience, we include a different (and short) proof:

Lemma 5.1. Let $G$ be a locally compact group. Then we have

$$
\tilde{\hat{\theta}}(\phi)=\hat{\theta}(\phi) \quad(\phi \in A(G)) .
$$

Proof. Let $\phi \in A(G)$, and note that both $\tilde{\hat{\theta}}(\phi)$ and $\hat{\theta}(\phi)$ are normal, which follows easily from the definition (see also $[\mathrm{N}-\mathrm{R}-\mathrm{S}$, Theorem 4.3] for $\hat{\theta}(\phi)$ ).

Both $\tilde{\hat{\theta}}(\phi)$ and $\hat{\theta}(\phi)$ are $L^{\infty}(G)$-bimodule maps: we already know this for $\hat{\theta}(\phi)$, and it follows for $\tilde{\hat{\theta}}(\phi)$ from $[\mathrm{P}-\mathrm{R}$, Theorem 2.3]. Furthermore, $\tilde{\hat{\theta}}(\phi)$ and $\hat{\theta}(\phi)$ coincide on all operators $\lambda(x)$ with $x \in G$ : we know that $\hat{\theta}(\phi)$ on $\operatorname{VN}(G)$ is nothing but the canonical action of $\phi$ on $\operatorname{VN}(G)$, and the same follows for $\tilde{\hat{\theta}}(\phi)$ from its definition. Consequently, we have

$$
\begin{aligned}
\tilde{\hat{\theta}}(\phi)\left(M_{f} \lambda(x)\right)=M_{f} \tilde{\hat{\theta}}(\phi) & (\lambda(x)) \\
& =M_{f} \hat{\theta}(\phi)(\lambda(x))=\hat{\theta}(\phi)\left(M_{f} \lambda(x)\right) \quad\left(f \in L^{\infty}(G), x \in G\right) .
\end{aligned}
$$

As in the proof of Proposition 4.2. we conclude that $\tilde{\hat{\theta}}(\phi)=\hat{\theta}(\phi)$.

Following $\mathrm{C}-\mathrm{L}$, where the analogous questions for $\mathrm{VN}(G)$ and $\mathcal{H}_{\sigma}$ were considered, we now study the pre-annihilator of $\tilde{\mathcal{H}}_{\sigma}$ in $\mathcal{T}\left(L^{2}(G)\right)$ for any $\sigma \in \mathcal{M}_{\mathrm{cb}}(A(G))$. 
Lemma 5.2. For any locally compact group $G$, we have

$$
T \cdot \omega=\hat{\theta}(\pi(\omega))(T) \quad\left(\omega \in \mathcal{T}\left(L^{2}(G)\right), T \in \mathcal{B}\left(L^{2}(G)\right) .\right.
$$

where $\pi: \mathcal{T}\left(L^{2}(G)\right) \rightarrow A(G)$ is the canonical quotient map.

Proof. Let $\omega \in \mathcal{T}\left(L^{2}(G)\right)$, and let $T \in \mathcal{B}\left(L^{2}(G)\right)$. Then we have

$$
\langle\rho, T \cdot \omega\rangle=\langle\omega \bullet \rho, T\rangle=\langle\omega, \rho \cdot T\rangle \quad\left(\rho \in \mathcal{T}\left(L^{2}(G)\right)\right) .
$$

Since $\rho \cdot T \in \operatorname{VN}(G)$ for $\rho \in \mathcal{T}\left(L^{2}(G)\right)$, the evaluation of $\omega$ at $\rho \cdot T$ depends only on $\pi(\omega)$, so that $\langle\rho, T \cdot \omega\rangle=\langle\pi(\omega), \rho \cdot T\rangle$ for each $\rho \in \mathcal{T}\left(L^{2}(G)\right)$. Hence we obtain

$$
\begin{aligned}
\langle\rho, T \cdot \omega\rangle & =\langle\pi(\omega), \rho \cdot T\rangle \\
& =\langle\pi(\omega), \hat{\mathcal{S}}(\rho \otimes T)\rangle \\
& =\left\langle\rho \otimes T, \hat{\mathcal{S}}^{*}(\pi(\omega))\right\rangle \\
& =\langle\rho \otimes T, \hat{\hat{\theta}}(\pi(\omega))\rangle \\
& =\langle\rho, \hat{\hat{\theta}}(\pi(\omega))(T)\rangle \\
& =\langle\rho, \hat{\theta}(\pi(\omega))(T)\rangle, \quad \text { by Lemma 5.1, }
\end{aligned}
$$

for all $\rho \in \mathcal{T}\left(L^{2}(G)\right)$, as desired.

The following is a consequence of $\left[\mathrm{P}-\mathrm{R}\right.$, Lemma 2.1], using the fact that $\left.\tilde{\hat{\theta}}\right|_{\mathcal{M}_{\mathrm{cb}}((A(G))}=$ $\hat{\theta}$. We include our own (short) proof for the sake of completeness:

Lemma 5.3. For any locally compact group $G$, we have

$$
\hat{\theta}(\phi)(\omega \cdot T)=\omega \cdot \hat{\theta}(\phi)(T) \quad\left(\phi \in \mathcal{M}_{\mathrm{cb}}\left((A(G)), \omega \in \mathcal{T}\left(L^{2}(G)\right), T \in \mathcal{B}\left(L^{2}(G)\right)\right) .\right.
$$

Proof. Let $\phi \in \mathcal{M}_{\mathrm{cb}}\left((A(G))\right.$, let $\omega, \rho \in \mathcal{T}\left(L^{2}(G)\right)$, and let $T \in \mathcal{B}\left(L^{2}(G)\right)$.

We first note that

$$
\begin{aligned}
\left\langle\pi\left(\hat{\theta}(\phi)_{*}(\rho)\right), S\right\rangle & =\left\langle\hat{\theta}(\phi)_{*}(\rho), S\right\rangle \\
& =\langle\rho, \hat{\theta}(\phi)(S)\rangle \\
& =\langle\rho, \phi \cdot S\rangle \\
& =\langle\pi(\rho), \phi \cdot S\rangle \\
& =\langle\pi(\rho) \phi, S\rangle \quad(S \in \mathrm{VN}(G)),
\end{aligned}
$$

so that

$$
\pi\left(\hat{\theta}(\phi)_{*}(\rho)\right)=\pi(\rho) \phi
$$


Now we obtain:

$$
\begin{aligned}
\langle\rho, \hat{\theta}(\phi)(\omega \cdot T)\rangle & =\left\langle\hat{\theta}(\phi)_{*}(\rho), \omega \cdot T\right\rangle \\
& =\left\langle\hat{\theta}(\phi)_{*}(\rho) \bullet \omega, T\right\rangle \\
& =\left\langle\omega, T \cdot \hat{\theta}(\phi)_{*}(\rho)\right\rangle \\
& =\left\langle\omega, \hat{\theta}\left(\pi\left(\hat{\theta}(\phi)_{*}(\rho)\right)\right)(T)\right\rangle, \quad \text { by Lemma [5.2. } \\
& =\langle\omega, \hat{\theta}(\pi(\rho) \phi)(T)\rangle, \quad \text { by (11), } \\
& =\langle\omega, \hat{\theta}(\pi(\rho))(\hat{\theta}(\phi)(T))\rangle \\
& =\langle\omega, \hat{\theta}(\phi)(T) \cdot \rho\rangle, \quad \text { by Lemma [5.2 again, } \\
& =\langle\rho \bullet \omega, \hat{\theta}(\phi)(T)\rangle \\
& =\langle\rho, \omega \cdot \hat{\theta}(\phi)(T)\rangle .
\end{aligned}
$$

Since $\rho \in \mathcal{T}\left(L^{2}(G)\right)$ is arbitrary, this yields that

$$
\hat{\theta}(\phi)(\omega \cdot T)=\omega \cdot \hat{\theta}(\phi)(T),
$$

as claimed.

Theorem 5.4. Let $G$ be a locally compact group, and let $\sigma \in \mathcal{M}_{\mathrm{cb}}(A(G))$. Then

$$
\tilde{I}_{\sigma}:=\overline{\left\{\hat{\theta}(\sigma)_{*}(\omega)-\omega: \omega \in \mathcal{T}\left(L^{2}(G)\right)\right\}} \mathcal{T}\left(L^{2}(G)\right)
$$

is the pre-annihilator of $\tilde{\mathcal{H}}_{\sigma}$ in $\mathcal{T}\left(L^{2}(G)\right)$ - so that $\tilde{\mathcal{H}}_{\sigma} \cong\left(\mathcal{T}\left(L^{2}(G)\right) / \tilde{I}_{\sigma}\right)^{*}$-and a two-sided ideal of $\left(\mathcal{T}\left(L^{2}(G)\right), \bullet\right)$.

Proof. It is straightforward to see that

$$
\omega \in \tilde{I}_{\sigma} \quad \Longleftrightarrow \quad\langle\omega, T\rangle=0 \text { for all } T \in \tilde{\mathcal{H}}_{\sigma} \quad\left(\omega \in \mathcal{T}\left(L^{2}(G)\right)\right)
$$

Hence, $\tilde{I}_{\sigma}$ is indeed the pre-annihilator of $\tilde{\mathcal{H}}_{\sigma}$ in $\mathcal{T}\left(L^{2}(G)\right)$, and $\left(\mathcal{T}\left(L^{2}(G)\right) / \tilde{I}_{\sigma}\right)^{*}=\tilde{I}_{\sigma}^{\perp} \cong$ $\tilde{\mathcal{H}}_{\sigma}$ holds.

We first show that $\tilde{I}_{\sigma}$ is a left ideal in $\mathcal{T}\left(L^{2}(G)\right)$. To this end, let $\omega, \rho \in \mathcal{T}\left(L^{2}(G)\right)$, and note that

$$
\begin{aligned}
\left\langle\omega \bullet \hat{\theta}(\sigma)_{*}(\rho), T\right\rangle & =\left\langle\hat{\theta}(\sigma)_{*}(\rho), T \cdot \omega\right\rangle \\
& =\langle\rho, \hat{\theta}(\sigma)(T \cdot \omega)\rangle \\
& =\langle\rho, \hat{\theta}(\sigma)(\hat{\theta}(\pi(\omega))(T))\rangle, \quad \text { by Lemma 5.2. } \\
& =\langle\rho, \hat{\theta}(\pi(\omega) \sigma)(T)\rangle \\
& =\langle\rho, \hat{\theta}(\pi(\omega))(\hat{\theta}(\sigma)(T))\rangle \\
& =\langle\rho, \hat{\theta}(\sigma)(T) \cdot \omega\rangle, \quad \text { again by Lemma 5.2, } \\
& =\langle\omega \bullet \rho, \hat{\theta}(\sigma)(T)\rangle \quad \\
& =\left\langle\hat{\theta}(\sigma)_{*}(\omega \bullet \rho), T\right\rangle . \quad\left(T \in \mathcal{B}\left(L^{2}(G)\right)\right),
\end{aligned}
$$


so that

$$
\omega \bullet \hat{\theta}(\sigma)_{*}(\rho)=\hat{\theta}(\sigma)_{*}(\omega \bullet \rho)
$$

and therefore

$$
\omega \bullet\left(\hat{\theta}(\sigma)_{*}(\rho)-\rho\right)=\omega \bullet \hat{\theta}(\sigma)_{*}(\rho)-\omega \bullet \rho=\hat{\theta}(\sigma)_{*}(\omega \bullet \rho)-\omega \bullet \rho \in \tilde{I}_{\sigma}
$$

Since $\omega, \rho \in \mathcal{T}\left(L^{2}(G)\right)$ were arbitrary, it follows that $\tilde{I}_{\sigma}$ is a left ideal as claimed.

To see that $\tilde{I}_{\sigma}$ is also a right ideal, let $\omega, \rho \in \mathcal{T}\left(L^{2}(G)\right)$, so that

$$
\begin{array}{rlr}
\left\langle\left(\hat{\theta}(\sigma)_{*}(\rho)\right) \bullet \omega, T\right\rangle & =\left\langle\left(\hat{\theta}(\sigma)_{*}(\rho)\right), \omega \cdot T\right\rangle \\
& =\langle\rho, \hat{\theta}(\sigma)(\omega \cdot T)\rangle \\
& =\langle\rho, \omega \cdot \hat{\theta}(\sigma)(T)\rangle, \quad \text { by Lemma 5.3. } \\
& =\langle\rho \bullet \omega, \hat{\theta}(\sigma)(T)\rangle \\
& =\left\langle\hat{\theta}(\sigma)_{*}(\rho \bullet \omega), T\right\rangle \quad\left(T \in \mathcal{B}\left(L^{2}(G)\right)\right)
\end{array}
$$

and thus

$$
\left(\hat{\theta}(\sigma)_{*}(\rho)\right) \bullet \omega=\hat{\theta}(\sigma)_{*}(\rho \bullet \omega) .
$$

A calculation similar to (12) then lets us conclude that $\tilde{I}_{\sigma}$ is a right ideal, too.

Remark. The above result mirrors both [C-L, Definition 3.2.1] and [J-N], Proposition 3.3] in our setting.

Finally, we consider another ideal of $\left(\mathcal{T}\left(L^{2}(G)\right), \bullet\right)$.

Proposition 5.5. Let $G$ be a locally compact group, and let $\sigma \in \mathcal{M}_{\mathrm{cb}}(A(G))$. Then

$$
L^{\infty}(G)_{\perp}:=\left\{\omega \in \mathcal{T}\left(L^{2}(G)\right):\left\langle\omega, M_{\phi}\right\rangle=0 \text { for all } \phi \in L^{\infty}(G)\right\}
$$

is a two-sided ideal in $\left(\mathcal{T}\left(L^{2}(G)\right), \bullet\right)$ which is contained in the augmentation ideal

$$
\mathcal{T}_{0}\left(L^{2}(G)\right):=\left\{\omega \in \mathcal{T}\left(L^{2}(G)\right):\langle\omega, 1\rangle=0\right\}
$$

and, if $\sigma(e)=1$, contains $\tilde{I}_{\sigma}$.

Proof. Trivially, $L^{\infty}(G)_{\perp} \subset \mathcal{T}_{0}\left(L^{2}(G)\right)$ holds.

Moreover, if $\sigma(e)=1$, then

$$
\left\langle\hat{\theta}(\sigma)_{*}(\rho)-\rho, M_{\phi}\right\rangle=\left\langle\rho, \hat{\theta}(\sigma)\left(M_{\phi}\right)\right\rangle-\left\langle\rho, M_{\phi}\right\rangle=\left\langle\rho, M_{\phi}\right\rangle-\left\langle\rho, M_{\phi}\right\rangle
$$

holds for all $\rho \in \mathcal{T}\left(L^{2}(G)\right)$ and $\phi \in L^{\infty}(G)$, so that $\tilde{I}_{\sigma} \subset L^{\infty}(G)_{\perp}$.

It remains to be shown that $L^{\infty}(G)_{\perp}$ is indeed an ideal of $\mathcal{T}\left(L^{2}(G)\right)$. 
Let $\rho, \omega \in \mathcal{T}\left(L^{2}(G)\right)$. Then we see that

$$
\begin{aligned}
\left\langle\rho \bullet \omega, M_{\phi}\right\rangle & =\left\langle\omega, M_{\phi} \cdot \rho\right\rangle \\
& =\left\langle\omega, \hat{\theta}(\pi(\rho))\left(M_{\phi}\right)\right\rangle, \quad \text { by Lemma [5.2. } \\
& =\left\langle\omega, M_{\phi} \hat{\theta}(\pi(\rho))(1)\right\rangle \\
& =\left\langle\omega, M_{\phi}(\pi(\rho) \cdot 1)\right\rangle \\
& =\langle\rho, 1\rangle\left\langle\omega, M_{\phi}\right\rangle \quad\left(\phi \in L^{\infty}(G)\right)
\end{aligned}
$$

holds. From (13), it is immediate that $L^{\infty}(G)_{\perp}$ is indeed a two-sided ideal of $\mathcal{T}\left(L^{2}(G)\right)$.

Remark. Since $L^{\infty}(G)_{\perp}$ is a two-sided ideal of $\left(\mathcal{T}\left(L^{2}(G)\right), \bullet\right)$, the product $\bullet$ induces a product - likewise denoted by •- on the quotient algebra $\mathcal{T}\left(L^{2}(G)\right) / L^{\infty}(G)_{\perp} \cong L^{1}(G)$. This product, however, is not the usual convolution product on $L^{1}(G)$ : from (13), it is clear that

$$
f \bullet g=\langle f, 1\rangle g \quad\left(f, g \in L^{1}(G)\right)
$$

\section{References}

[Aze] R. Azencott, Espaces de Poisson des groupes localement compacts. Lecture Notes in Mathematics 148, Springer-Verlag, 1970.

[C-L] C.-H. Chu and A. T.-M. LAU, Harmonic Functions on Groups and Fourier Algebras, Lecture Notes in Mathematics 1782. Springer-Verlag, Berlin, 2002.

[dC-H] J. De Cannière and U. HaAgerup, Multipliers of the Fourier algebras of some simple Lie groups and their discrete subgroups. Amer. J. Math. 107 (1985), 455-500.

[E-R] E. G. Effros and Z.-J. Ruan, Operator Spaces. Clarendon Press, 2000.

[E-S] M. Enock and J.-M. Schwartz, Kac Algebras and Duality of Locally Compact Groups. Springer Verlag, 1992.

[Eym] P. Eymard, L'algèbre de Fourier d'un groupe localement compact. Bull. Soc. Math. France 92 (1964) 181-236.

[For] B. E. Forrest, Fourier analysis on coset spaces. Rocky Mountain J. Math. 28 (1998), 173-190.

[F-R-S] B. E. Forrest, V. Runde, and N. Spronk, Operator amenability of the Fourier algebra in the cb-multiplier norm. Canadian J. Math. (to appear).

[Fur] H. Furstenberg, Boundary theory and stochastic processes on homogeneous spaces. In: Harmonic Analysis on Homogeneous Spaces, pp. 193-229. Proceedings of Symposia in Pure Mathematics 26, American Mathematical Society, 1973.

[Gha] F. Ghahramani, Isometric representation of $M(G)$ on $B(H)$. Glasgow Math. J. 23 (1982), 119-122. 
[G-D] A. Granas and J. Dugundu, Fixed Point Theory. Springer-Verlag, 2003.

[H-R $\quad$ E. Hewitt and K. A. Ross, Abstract Harmonic Analysis, I (2 ${ }^{\text {nd }}$ edition). Grundlehren der Mathematischen Wissenschaften 115, Springer-Verlag, 1979.

[H-K] U. HaAgerup and J. Kraus, Approximation properties for group $C^{*}$-algebras and group von Neumann algebras. Trans. Amer. Math. Soc. 344 (1994), 667-699.

[Izu] M. Izumi, Non-commutative Poisson boundaries. In: M. Kotani, T. Shirai, and T. Sunada (ed.s), Discrete Geometric Analysis, pp. 69-81. Contemporary Mathematics 347, American Mathematical Society, 2004.

$[\mathrm{J}-\mathrm{N}] \quad$ W. Jaworski and M. Neufang, The Choquet-Deny equation in a Banach space. Canadian J. Math. (to appear).

[Jol] P. Jolissaint, A characterization of completely bounded multipliers of Fourier algebras. Colloq. Math. LXIII (1992), 311-313.

[K-L] E. Kaniuth and A. T.-M. LaU, A separation property of positive definite functions on locally compact groups and applications to Fourier algebras. J. Funct. Anal. 175 (2000), 89-110.

[Lau] A. T.-M. LAU, Invariantly complemented subspaces of $L_{\infty}(G)$ and amenable locally compact groups. Illinois. J. Math. 26 (1982), 226-235.

[Neu 1] M. Neufang, Abstrakte Harmonische Analyse und Modulhomomorphismen über von Neumann-Algebren. PhD thesis, Universität des Saarlandes, 2000.

[Neu 2] M. Neufang, Isometric representations of convolution algebras as completely bounded module homomorphisms and a characterization of the measure algebra. Preprint.

[Neu 3] M. Neufang, A quantized analogue of the convolution algebra $L_{1}(G)$. Preprint.

[N-R-S] M. Neufang, Z.-J. Ruan, and N. Spronk, Completely isometric representations of $M_{c b} A(G)$ and $U C B(\hat{G})^{*}$. Trans. Amer. Math. Soc. (to appear).

[Nev] J. Neveu, Mathematical Foundations of the Calculus of Probability. Holden-Day, 1965.

[Pir] A. Pirkovskir, Biprojectivity and biflatness for convolution algebras of nuclear operators. Canadian Math. Bull. 47 (2004), 445-455.

$[\mathrm{P}-\mathrm{R}] \quad$ A.-M. Popa and Z.-J. Ruan, Uniformly complete quotient space UCQ $(G)$ and completely isometric representations of $\operatorname{UCQ}(G)^{*}$ on $\mathcal{B}\left(L_{2}(G)\right)$. Proc. Amer. Math. Soc. (to appear).

[Rev] D. Revuz, Markov Chains. North Holland, 1984.

[Spr] N. Spronk, Measurable Schur multipliers and completely bounded multipliers of the Fourier algebras. Proc. London Math. Soc. 89 (2004), 161-192

[Stø] E. Størmer, Regular abelian Banach algebras of linear maps of operator algebras. $J$. Funct. Anal. 37 (1980), 331-373.

[T-T 1] M. Takesaki and N. Tatsuuma, Duality and subgroups. Ann. of Math. 93 (1971), $344-364$.

[T-T 2] M. Takesaki and N. Tatsuuma, Duality and subgroups, II. J. Funct. Anal. 11 (1972), 184-190. 
[Wil] G. A. Willis, Probability measures on groups and some related ideals in group algebras. J. Funct. Anal. 92 (1990), 202-263.

First author's address: School of Mathematics and Statistics Carleton University

Ottawa, Ontario

Canada K1S 5B6

E-mail: $\quad$ mneufang@math.carleton.ca

URL: $\quad$ http://mathstat.carleton.ca/ $\quad$ mneufang/

Second author's address: Department of Mathematical and Statistical Sciences

University of Alberta

Edmonton, Alberta

Canada T6G 2G1

E-mail: $\quad$ vrunde@ualberta.ca

URL: $\quad$ http://www.math.ualberta.ca/ $\sim$ runde/ 